தொல்காப்பிய மெய்ப்பாடுகளின்

உள்ளீடுகளும் நற்றிணை

OPEN ACCESS

உளவியலும்

மு. இளங்கோவன் அ, *

அ தமிழ் உயராய்வு மையம், செந்தமிழ் கல்லூரி, மதுரை-625001, தமிழ்நாடு, இந்தியா.

\title{
Tolkappiya Meyppatukalin ullitukalum Natrinai Mantarin ulaviyalum
}

\author{
M. Elangovan ${ }^{a}{ }^{*}$ \\ a Department of Tamil, Senthamil College, Madurai-625001, Tamil Nadu, India
}

* Corresponding Author: ilangoilango566@gmail.com

Received: 23-03-2021

Revised: 16-01-2022

Accepted: 18-01-2022

Published: 28-02-2022

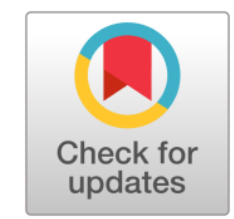

\begin{abstract}
Tholkappiyam is the most ancient extent Tamil grammer text and the oldest extant long work of Tamil Literature. The surviving manuscripts of the Tholka ppiyam consists of three books each with nine chapters namely Eluthu, Chol and porul. Among the nine chapters in porul, Maippattiyal is one of the above nine. It is named thus in view of the fact that it expounds the feelings and emotions. The scholars will say the eight states of being beginning with Nagai, Azukai, ilivaral, Marutkkai, Acham, Perumitham, Vekuli, uvakai are the basic eight categories of Meyppadu. This Article Compares the above eight categories with Natrinai is a book of Ettuthogai a Sangam literature anthology. This belongs to Sangam Poetry of the oldest extant Tamil Literature.
\end{abstract}

Keywords: Tholkappiyam, Tamil Grammer, Natrinai, Tamil Literature.

\section{முன்னுரை}

மெய்ப்பாடு என்பது பொருட்பாடு, சத்துவம், ரஸம், விறல், சுவை எனவும் அழைக்கப்படும். நாவால் தோன்றும் சுவை ஆறாகும். அதனை அறுசுவை என்பர். மெய்யால் (உடல்) தோன்றும் சுவை எட்டாகும். அதனை எண்சுவை என்பர். நாவால் தோன்றும் சுவையை, சுவைப்போன் உணர முடியும். மெய்யால் தோன்றும் சுவையை அவனைக் காண்போன் அறியமுடியும். நாவின் சுவை, சுவைப்போனுக்கு மகிழ்வைத்தரும். எண்சுவை, அதனைப் புலப்படுத்துவோனை உற்று நோக்குவோனுக்கு மகிழ்வைத்தரும். இம்மெய்ப்பாடு, உளவியல் துறையோடு தொடர்புடையது. உளவியல், மனித வாழ்வோடு தொடர்புடையது. இவ்உளவியல், மனித நடத்தையின் அடிப்படைச் செயல்பாடுகளை விளக்கும் ஆற்றலுடையது. உளவியல் என்பது மனத்தைப் பற்றிய அறிவியல்துறையாகும். மனம் என்பதைனை உடலின் பிற உறுப்புகளைப்போலக் கண்ணால் காண இயலாது. பழந்தமிழரின் மனம், உளவியல் பாங்குடன் தொல்காப்பிய மெயப்பாடுகள் வழியே நற்றிணைப் பாடல்கள் மூலம் எவ்வாறு புலப்படுத்தப்படுகின்றன என்பதனை இக்கட்டுரை விவரிக்கின்றது (Ilampuranar, 1953; Parimanam \& Balasubramaniyam, 2004).

\section{நகை}

நகை என்பது சிரிப்பு, புன்னகை, ஹாஸ்யம் எனவும் அழைக்கப்படும். இம்மெய்ப்பாடு, தொல்காப்பியர் கூறிய எட்டனுள் முதலாவதாகும் (Ilampuranar, 1969). மனத்தினுள் தோன்றிய மகிழ்ச்சிக் குறிப்பை மற்றவர்க்கு முகக்குறிப்பால் இம்மெய்ப்பாடு வெளிப்படுத்தும். 
தலைவியின் காமநோய் தலைவனால் வந்தது. இதை அறியாத தாய் இது முருகக்கடவுளால் வந்தது என்று கருதி வேலனைக் (பூசாரி) கொண்டு வெறியாடலுக்கு (பூசைக்கு) ஏற்பாடு செய்தாள். அப்போது தோழி, வேலனிடம், நீ இதனை அறியாதிருந்தால் (தலைவியின் காமநோயை) உண்மையில் நீ அறிவற்றவனே ஆவாய் என்கின்றாள்.

தாய், தலைவிக்கு நோய்வர கடவுள் காரணம் என எண்ணினாள். ஆனால், உண்மைக்காரணம் தலைவன் என்பதைத் தோழி அறிவாள். தோழி, வேலனிடம் உரைத்தது இங்கு தலைவன்மீது ஏற்றிக் கூறப்படுகின்றது. தோழி, வேலனிடம் உரைப்பது போலத் தலைவனிடம், நீ கடவுளர்களில் ஒருவனாகக் கருதப்பெறுவதற்கு உரியவன் ஆயினும் ஆகுக் உறுதியாக நீ அறிவற்றவனே ஆவாய் என்கின்றாள். தாயின் அறியாமையையும் தோழியின் அறிவையும் நகையுடன் புலப்படுத்தும் நற்றிணை அடிகள்,

“கார் நறுங் கடம்பின் கண்ணி சூடி

வேலன் வேண்ட வெறி மனைவந்தோய்

கடவுள் ஆயினும் ஆக

மடவை மன்ற வாழிய முருகே"

என்பதாகும் (Ilampuranar, 1969). இது, தொல்காப்பியர் மெய்ப்பாட்டியலுள்,

“எள்ளல் இளமை பேதைமை மடனென்று

உள்ளப் பட்ட நகைநான் கென்ப"

என நகைக்குக் கூறிய நான்கு நிலைக்களன்களுள் எள்ளல் குறித்து எழுந்த நகையாகும் (llampuranar, 1953). எள்ளல், தான் பிறரை எள்ளி நகுதலும் பிறரால் எள்ளப்பட்டவழித் தான் நகுதலும் என இரண்டு வகைப்படும். இது, தோழி தலைவனை எள்ளி நகுவதால், 'தான் பிறரை எள்ளி நகுதல்' எனும் வகையுள் அடங்கும். இறைநம்பிக்கையால் நோய் நீங்கும் எனும் பழந்தமிழர் உளவியல் இதனால் புலப்படும்.

\section{அழுகை}

அழுகை என்பது அவலம் எனவும் அழைக்கப்படும். இம்மெய்ப்பாடு தொல்காப்பியர் கூறிய எட்டனுள் இரண்டாவதாகும். உள்ளத்தின் சோகத்தைக் கண்ணீராலோ சொல் முதலிய பிற குறிப்புகளாலோ இம்மெய்ப்பாடு வெளிப்படுத்தும். இது, தானே அவலித்தலும் பிறர் அவலம் கண்டு அவலித்தலும் என இருவகையாகும். பிறர் அவலம் கண்டு அவலித்தலை நற்றிணைப்பாட்டு ஒன்றால் அறியலாம்.

காட்டு வழியாகச் செல்லும் மக்களின் தலைகளைப் புலிக்குட்டிகள் மோதும். அதனால், அக்குட்டிகளின் தலைகளில் சிவப்புக்கறை (இரத்தக்கறை) படியும் வாயெல்லாம் குருதி படிந்திருக்கும். மாலைப்பொழுதில் பெரியதலைகளை உடையப் புலிக்குட்டிகள் தாம் பதுங்கி இருக்கும் மரங்களின் தூறுகளை நிமிர்ந்து பார்க்கும் இத்தகையக் காட்டில் இரவுப்பொழுதில் கூர்மையான பற்களை உடைய மென்மைத்தன்மை வாய்ந்த பெண்ணை, முன்செல்லவிட்டுப் பின்தொடரும் இந்த இளையவன் உள்ளம் காற்றோடு கலந்து பெய்யும் மழையில் பெரிய கற்களைப் புரட்டுமாறு இடிக்கும் இடியைவிடக் கொடியது. இச்சூழலை உடைய காட்டின் வழியே தலைவனும் தலைவியும் சென்றதைக் கண்டோர்,

“வை எயிற்று ஐயள் மடந்தைமுன் உற்று

எல்லிடை நீங்கும் இளையோன் உள்ளம்

காலொடு பட்ட மாரி

மால் வரை மிளிர்க்கும் உருமினும் கொடிதே" 
எனக்கூறுவதை நற்றிணைப் பதிவு செய்துள்ளது (Ilampuranar, 1953). இப்பாட்டு, தொல்காப்பியர், மெய்ப்பாட்டியலுள்,

“இளிவே இழவே அசைவே வறுமையென

விளிவில் கொள்கை அழுகை நான்கே”

என அழுகைக்குக் கூறிய நான்கு நிலைக்களன்களுள் இளிவு என்பதனுள் அடங்கும். இளிவு என்பதனை (Ilampuranar, 1953) இழவு என்பர். இளிவு என்பது பிறரால் இகழப்பட்டு எளியனாதல் என விளக்கப்படும். மேற்கூறிய நற்றிணைப் பாட்டில் தலைவன் உள்ளம் கண்டு, கண்டோர் இகழ்வது கூறப்படுகின்றது. தலைவி நடக்க இயலாத தன்மை உடையவள் என்பதையும் அவளுடைய நடையையும் பின்னழகையும் கண்டு மகிழும் போக்கினையும் அவளுக்குப் பாதுகாப்புத் தந்துப் பின் செல்கின்றான் என்பதையும் இப்பகுதி உளவியல் பாங்கில் உணர்த்துகின்றது.

\section{இளிவரல்}

இளிவரல் என்பது இழிவு எனவும் அழைக்கப்படும். இம்மெய்ப்பாடு, தொல்காப்பியர் கூறிய எட்டனுள் மூன்றாவதாகும். பிறரால் வருந்தி இகழப்படும்போது இம்மெய்ப்பாடு வெளிப்படும். முன்னர் ஒருசமயம் தலைவியைப் பிரிந்து பொருள் தேடும் பொருட்டுச் சென்ற தலைவன் மீண்டும் ஒருமுறை பொருள் தேடச் செல்லும்போது தன் நெஞ்சிற்கு வருந்திக் கூறுகின்றான். நெஞ்சமே! நம் தலைவி, மனைக்கு ஒளிதரும் விளக்கினை ஏற்றி வைத்து அதன்முன் நின்று நம்மை நினைத்து வருந்தும் மாலைப்பொழுது இதுவாகும். நானும் அவளை நினைத்து வருந்தினேன். இதனை, நற்றிணை,

"உள்ளினெ னல்லனோ, யானே - உள்ளிய

வினைமுடித் தன்ன இளியோள்

மனைமாண் சுடரொடு படர்பொழு தெனவே." (Ilampuranar, 1953)

எனக் கூறும் இப்பாட்டு, தொல்காப்பியர், மெய்ப்பாட்டியலுள்,

"மூப்பே பிணியே வருத்த மென்மையொடு

யாப்புற வந்த இளிவரல் நான்கே"

என இளிவரலுக்குக் கூறிய நான்கு நிலைக்களன்களுள் வருத்தம் என்பதனுள் அடங்கும். வருத்தம் என்பது தனக்காகவும் பிறருக்காகவும் வருத்தம் கொள்ளுதல் ஆகும். இப்பாட்டில், தலைவன் தனக்காகவும் தலைவிக்காகவும் வருந்துகின்றான். தலைவன், தலைவியை நினைத்து வருந்தியிருக்க, இனி எவ்வாறு அவளைப் பிரிவேன் என வருந்தித் தன் எண்ணத்தை மாற்றிக் கொண்ட உளவியற்பாங்கு தெரிகின்றது (Balasubramanian, 2006).

\section{மருட்கை}

மருட்கை என்பது வியப்பு, ஆச்சரியம் எனவும் அழைக்கப்படும். இது, தொல்காப்பியர் கூறிய எட்டனுள் நான்காம் மெய்ப்பாடு ஆகும். உலக வழக்கத்தில் நிகழாது காண்போர் வியக்கும் வகையில் மாறுபட்டு நிகழும்போது இம்மெய்ப்பாடு நிகழும்.

குளிர்ந்த கண்களும், தழைகள் கொண்ட பூக்களால் ஆன உடையும், அசையும் அல்குல் தடமும், நீலமணிகள் தொடுக்கப்பெற்ற அணிகலனும் மேனியில் விளங்கத் திகழும் இவ்இளம் மங்கை யாருடைய மகள் எனத் தலைவன் தன் நெஞ்சிற்குச் சொல்லி வியப்பதை,

“அல்குபடர் உழந்த அரிமதர் மழைக்கண்

பல்பூம் பகைத்தழை நுடங்கும் அல்குல் 
திருமணி புரையும் மேனி மடவோள்"

எனும் நற்றிணைப் பாட்டால் அறியலாம் (Ilampuranar, 1953). இப்பாட்டு, தொல்காப்பியர், மெய்ப்பாட்டியலுள்,

"புதுமை பெருமை சிறுமை ஆக்கமொடு

மதிமை சாலா மருட்கை நான்கே"

என மருட்கைக்குக் கூறிய நான்கு நிலைக்களன்களுள் புதுமை என்பதனுள் அடங்கும். புதுமை என்பது ஏதாவது ஒன்றின்மூலம் ஏதாவது ஓர் இடத்தில் முன்எப்பொழுதும் காணப்படாத நிலை கண்டு வியத்தலாகும். இப்பாட்டில், இயற்கைப் புணர்ச்சியில் தலைவியைக் கண்ட தலைவன் அவள்மீது தன் நெஞ்சைப் பறிகொடுத்து காதல் கொண்ட உளவியல் புலப்படுகின்றது.

\section{அச்சம்}

அச்சம் என்பது பயம் எனவும் அழைக்கப்படும். இது, தொல்காப்பியர் கூறிய எட்டனுள் ஐந்தாம் மெய்ப்பாடு ஆகும். காணத்தகாததைக் காணும்போதும் எண்ணத்தகாததை எண்ணும் போதும் கேட்கத்தகாததைக் கேட்கும்போதும் இவைபோன்ற பிறவற்றாலும் இம்மெய்ப்பாடு நிகழும்.

தலைவன் நாட்டின் மலை உயிர்களைக் கொல்லும் அச்சம் தரும் ஆண்புலி, பெண் யானை கலங்குமாறு அதன் துணையாகிய ஆண்யானையினைக் கொல்லும் என அஞ்சும் தன்மையினை உடையதாகும். தலைவனுக்கு உணர்த்தும் வகையில் தோழி, தலைவியை நோக்கிக் கூறியதில் வெளிப்படும் இம்மெய்ப்பாட்டை, நற்றிணை,

“குறுங்கை இரும்புலிக் கோள்வல் ஏற்றை

பூநுதல் இரும்பிடி புலம்பத் தாக்கித்

தாழ்நீர் நனந்தலைப் பெருங்களிறு அடூஉம்

கல்லக வெற்பன்"

எனப்பாடும் (Ilampuranar, 1953). இப்பாட்டு, தொல்காப்பியர் மெயப்பாட்டியலுள்,

“அணங்கே விலங்கே கள்வர்தம் இறையெனப்

பிணங்கல் சாலா அச்சம் நான்கே”

என அச்சத்திற்குக் கூறிய நான்கு நிலைக்களன்களுள் விலங்கு என்பதனுள் அடங்கும். விலங்கு என்பது புலி, யானை முதலிய கொடிய விலங்குகளால் தோன்றும் அச்சம் எனும் மெய்ப்பாடாகும். புலி களிற்றைத் தாக்கிக் கொல்லும் வழி என்பதால் உற்றவர் பாதுகாப்பர் என்பது இதனுள் பொதிந்த உளவியற் கருத்தாகும். யானையைப் புலி தாக்கிக் கொல்வதால் தோன்றும் விலங்கு குறித்த அச்சத்தைக் குறுந்தொகை,

“நெடுங்கை வன்மான் கடும்பகை யுழந்த

குறுங்கை யிரும்புலிக் கோள்வ லேற்றை"

எனப்பாடும் 'Swaminatha Iyer, 2017).

\section{பெருமிதம்}

பெருமிதம் என்பது வீரம், ஆற்றல், பலம், வலிமை எனவும் அழைக்கப்படும். இப்பெருமிதம், இளம்பூரணரால், “எல்லாரொடும் ஒப்ப நில்லாது பேரெல்லையாக நிற்றல் பெருமிதம்" என விளக்கப்படும். இது, தொல்காப்பியர் கூறிய எட்டனுள் ஆறாம் மெய்ப்பாடு ஆகும். பிறரினும் உயர்ந்தும் சிறந்தும் தனித்தும் நிற்கையில் இம்மேம்பாடு வெளிப்படும். 
சோழமன்னர்கள் கொங்கு நாட்டினரைப் பணியச்செய்ய போஒர் என்னும் ஊரின் குறுநில மன்னனானப் பழையன் என்பான் மூலம் அவனது போர்யானைப்படைகளை ஏவினர். அப்படை

சோழர்களுக்கு வெற்றியைத் தந்தது. இவ்விருவரின் பெருமித மெய்ப்பாட்டை நற்றிணை,

“கொற்றச் சோழர் கொங்கர்ப் பணீஇயர்

வெண் கோட்டு யானைப் போஒர் கிழவோன்

பழையன் வேல் வாய்த்தன்ன"

எனப்பாடி வெளிப்படுத்துகின்றது ～(llampuranar, 1953). இப்பாட்டு, தொல்காப்பியர், மெய்ப்பாட்டியலுள்,

“கல்வி தறுகண் புகழ்மை கொடையெனச்

சொல்லப் பட்ட பெருமிதம் நான்கே"

எனப் பெருமிதத்திற்குக் கூறிய நான்கு நிலைகளன்களுள் தறுகண் என்பதனுள் அடங்கும். தறுகண் என்பது வீரமாகும். இங்கு சோழன் வீரமும் பழையன் வீரமும் சிறப்பிக்கப்படுகின்றது. இவ்வேற்படையைப் போலத் தப்பாத தலைவனின் நற்சொல்கேட்டுத்தலைவி தெளிந்தாள் என்பது இங்கு புலப்படும் உளவிற் கருத்தாகும். பழையன் வீரமும் அவன் ஊரும்,

“மாரி அம்பின் மழைத்தோள் பழையன்

காவிரி வைப்பின் போஒர்"

என அகநானூற்றால் சிறப்பிக்கப்படுதல் இங்கு நினைக்கத்தக்கதாகும் (Somasundranaar, 1970).

\section{வெகுளி}

வெகுளி என்பது உருத்திரம், பெருஞ்சினம், பெருங்கோபம் எனவும் அழைக்கப்படும். இம்மெய்ப்பாடு, தொல்காப்பியர் கூறிய எட்டனுள் ஏழாவதாகும். இது விரும்பத்தகாதனவற்றாலும் ஏற்கத்தகாதனவற்றாலும் இவைபோன்ற பிற்வற்றாலும் வெளிப்படும்.

தோழி, பாணனிடம் கூறுகின்றாள். தலைவன், காதில் குண்டலமும் கழுத்தில் மாலையும் கையில் வளையலும் அணிந்து பெண்வேடமிட்டுப் பரத்தையருடன் துணங்கைக் கூத்தாடினான். இதனால், சினங்கொண்டு, அவனை நாணமற்றவன் எனச் சினந்து கூறினேன். இப்பாட்டில் தோன்றும் வெகுளி எனும் மெய்ப்பாட்டை

“குழையன் கோதையன் குறும் பைந் தொடியன்

விழவு அயர் துணங்கை தழுகம் செல்ல

அதன் எதிர்

நாண் இலை எலுவ என்று வந்திசினே"

என நற்றிணைப் புலப்படுத்தும் (Balasubramanian, 2007). இது, தொல்காப்பியர்

“உறுப்பறை குடிகோள் அலைகொலை என்றன

வெறுப்ப வந்த வெகுளி நான்கே"

என வெகுளிக்குக் கூறிய நான்கு நிலைக்களன்களுள் குடிகோள் என்பதனுள் அடங்கும். குடிகோள் என்பது குடிப்பிறப்பிற்குக் கேடு சூழ்தல் ஆகும். இங்கு, தலைவன் தன்குடிப்பிறப்பிற்குக் கேடு சூழலும் விதத்தில் பரத்தையரோடு பெண்வேடமிட்டு ஆடியது சுட்டப்படுகின்றது (Ilampuranar, 1953). 
துணைங்கைக் கூத்தினைச் சிலப்பதிகாரம், இந்திரவிழா ஊர் எடுத்த காதை, “துணங்கையர் குரவையர் அணங்கு எழுந்து ஆடி" (Swaminatha lyer, 2013) என்கின்றது. மகளிர் இரு கைகளையும் மடக்கி அடித்து ஆடும் ஆட்டம் துணங்கை என்பதனைத் திவாகர நிகண்டு,

"முடக்கிய விருகை பழுப்புடை யொற்றித்

துடக்கிய நடையது துணங்கை ஆகும்"

எனப்பாடுதலால் அறியலாம் (Divakara Munivar, 1958). தலைவன் துணங்கை ஆடலை,

"முழாவிமிழ் துணங்கைக்குத் தழுஉப்புணை யாகச்

சிலைப்புவல் லேற்றிற் றலைக்கை"

எனப் பதிற்றுப்பத்து பாடுகின்றது. ஒவ்வொரு ஆண்மையும் பெண்மை உள்ளடக்கியது என்ற உளவியல் இப்பாட்டில் பொதிந்துள்ளது (Swaminatha Iyer, 2018).

\section{உவகை}

உவகை என்பது மகிழ்ச்சி, களிப்பு, ஆனந்தம் எனவும் அழைக்கப்படும். நகை என்பதற்கும் உவகை என்பதற்கும் வேறுபாடு என்னவென்றால் முன்னது மகிழ்வால் உண்டாகும் சிரிப்பை வெளிப்படப் புலப்படுத்துதலாகும். பின்னது மகிழ்வால் உண்டாகும் சிரிப்பை மனத்திற்குள் மட்டும் புலப்படுத்துதலாகும். இது, தொல்காப்பியர் கூறிய எட்டனுள் எட்டாம் மெய்ப்பாடு ஆகும்.

தலைவி தோழியிடம் தலைவன் குறித்துச் சொல்கின்றாள். என்தலைவன் சொன்ன சொல்லில் இருந்து மாறாத வாய்மையாளர், எல்லாக் காலத்திலும் இனிமையுடன் பழகும் பண்பாளர் என்னைவிட்டு எப்போதும் பிரியாத அன்பாளர். தலைவியின் உள்ளத்திற்குள் பூத்துப் பூரித்து வெளிப்படும் இவ்உவகை மெய்ப்பாட்டை,

“நின்ற சொல்லர் நீடு தோறு இனியர்

என்றும் என்தோள் பிரிபு அறியலரே

எனும் அடிகளில் காணலாம் (Ilampuranar,1953). இப்பாட்டு, தொல்காப்பியரின் மெய்ப்பாட்டியலுள்,

"செல்வம் புலனே புணர்வுவிளை யாட்டென

அல்லல் நீத்த உவகை நான்கே”

என உவகைக்குக் கூறிய நான்கனுள் புணர்வு என்பதனுள் அடங்கும். புணர்வு என்பது காமப்புணர்வாகும் (Ilampuranar, 1953). இப்பாட்டில், தலைவன் குறித்துத் தலைவியின் உள்ளத்துத் தோன்றும் உவகை மெய்ப்பாடு அவளது சொற்களால் புலப்படுகின்றது. தலைவி, தலைவனைப் புகழ்ந்து கூறியதால் அவன் அவளைப் பிரியக் கருதான் என்ற உளவியல் இதில் அமைந்துள்ளது.

\section{முடிவுரை}

தொல்காப்பியரின் மெய்ப்பாடுகள் நற்றிணையில் மெய்ம்மைப்பாடுகளாகப் புலனாகின்றன. மெய் (உடல்) உள்ள மனிதன் உருவம் உடையவன் ஆகின்றான். அவ்உருவத்தால் தோன்றும் மெய்ப்பாட்டு உணர்வோ அருவமாக (உருவம் அற்றதாக) இருக்கின்றது. அந்த அருவத்தைப் பருப்பொருளாக்கித் தருவதில் (காணக் கூடியதும் உணரக்கூடியதுமான பொருள்) நற்றிணை ஒரு நல்லிடத்தைப் பெற்றுள்ளது. நற்றிணையின் மெய்ப்பாடுகளைப் பயில்வோரின் உள்ளம் அவற்றை அருவமாகவே அறிந்துணர்கின்றது. உலகில் வாழும் எல்லா மக்களும் தத்தம் மனஉணர்வினை ஒரே மாதிரியான மெய்ப்பாடுகளாகவே ெளிப்படுத்துகின்றரர். இம்மெய்ப்பாடுகளையேத் தொல்காப்பியர் மெய்ப்பாட்டியலில் வெளிப்படுத்தி உள்ளார். தொல்காப்பியரின் வெளிப்பாடுகளையே நற்றிணை தன் புலப்பாடுகளாகக் கூறுகின்றது. 


\section{References}

Balasubramanian, K.V., (2006) Natrinai, New Century Book House, Chennai, India.

Divakara Munivar, (1958) Chendan Divakaram, Kazhaga veliyedu, Chennai, India.

Ilampuranar, (1953) Tholkaapiyam Porulathigaram, Kazhaga veliyedu, Chennai, India.

Ilampuranar, (1969) Tolkappiyam, The South India Saiva Siddhantha Works Publishing Society, Tinnevelly, India.

Parimanam, A.M., Balasubramaniyam, K.V., (2004) Natrinai, New Century Book House Pvt Ltd, Chennai, India

Somasundranaar, P.V., (1970) Agananooru, Kazhaga veliyedu, Chennai, India.

Swaminatha Iyer, U.V., (2013) Silapathigara Moolamum arumpathavuraiyum adiyaruku nallaraiyum, U.Ve.Sa, Library, Chennai, India.

Swaminatha Iyer, U.V., (2017) Kurunthogai, U.Ve.Sa, Library, Chennai, India.

Swaminatha Iyer, U.V., (2018) Pathitrupathu, U.Ve.Sa, Library, Chennai, India.

Funding: No funding was received for conducting this study.

Conflict of Interest: The Author has no conflicts of interest to declare that they are relevant to the content of this article.

About the License:

\section{(c)(1)}

Attribution 4.0 International (CC BY 4.0)
(C) The Author 2022. The text of this article is open access and licensed under a Creative Commons Attribution 4.0 International License 